\title{
Transformaciones recientes de la estructura socioclasista cubana ${ }^{1}$
}

\author{
M ayra Paula Espina Prieto
}

Centro de Investigaciones Psicológicas y Sociológicas (CIPS).

Calle B, \#352, esquina a 15. 10400 Vedado. La H abana. Cuba

Fecha de recepción: 1995

\section{Resumen}

Uno de los el ementos básicos para caracterizar una sociedad concreta es precisamente su estructura social, entendida como la segmentación en clases, capas y grupos - y las relaciones entre estos segmentos- que le es consustancial a su reproducción.

La revolución cubana significó un cambio radical en la composición socioclasista nacional, al sustituir aceleradamente la vieja estructura típica del capitalismo dependiente y periférico por la correspondiente a la transición socialista, caracterizada por al tos grados de igualdad e integración social.

El artículo ofrece una imagen de esa estructura propia de la transición, de sus debilidades y fortalezas y presenta al gunos de los problemas claves que ha hecho emerger la crisis cubana de los noventa. Intenta demostrar que con la crisis y la estrategia diseñada para su enfrentamiento ha aparecido una nueva etapa en la reproducción socioestructural, asociada fundamentalmente a la presencia de un nuevo modelo de organización del sistema de propiedad sobre los medios de producción, donde ha cambiado sustantivamente el papel del Estado en la configuración de esta estructrua y en el que las desigualdades social es y los procesos de desintegración tienen un espacio mayor.

Palabras clave: estructura socioclasista, desigualdades sociales, relaciones de propiedad, socialismo.

Abstract. Recent transformations of Cuban class structure

O ne of the basic elements of a specific society is ther social structure, that is, segmentation and differentiation by classes, groups and their relations with the social reproduction process.

Cuban revolution meant a radical change of the national class composition the old

1. Este texto se basa en los siguientes informes de investigación: Espina, M ;; N úñez, L.; M artín, L. y Blanco, J.M . (1989). «R eproducción socioclasista en Cuba». M ecanuscrito. Fondos del CIPS. Espina, M.; N úñez, L.; M artín, L.; Perera, M . (1995). « mpactos socioestructurales del reajuste económico». M ecanuscrito. Fondos del CIPS. Constituye además un avance del libro Crisis, reajuste y des gualdades sociales Un punto de vista sociológico sobrela sociedad cubana en los noventa, (título provisional). En preparación por la colección «Sociedad Cubana» de la Editorial Academia. 
social structure linked to the dependent and peripheral capitalism was quickly substituted by socialist transition in colsich high levels of social equality and integration were able.

The article clearly examines the transition social structure, its strong and weak elements and shows some key problems emerging from 1990 economic crisis. It tries to demonstrate that the crisis and its solution has devoeloped into a new steps of the social structural reproduction. This has been basically associated with the existence of a new model of the property system organization of the means of production.

The role of the state has accordingly changed and has influenced the system of social inequalities and the processes of non integration.

Key words: Cuban class structure, social inequalities, propiety relations, socialism.

\section{Sumario}

Introducción La etapa actual: la crisis, el reajuste económico y el nuevo modelo

Transición socialista y dinámica de los procesos socioestructurales

para la estructura social

\section{Introducción}

El concepto de «estructura social» resulta extremadamente amplio y hasta ambiguo, debido a los diferentes usos y significados que le han atribuido las ciencias sociales. Pero cuando lo asumimos desde la óptica de una sociología de las desigualdades sociales por lo general se utiliza para designar el conjunto de estratos, sectores, grupos y espacios sociales diferentes en que una sociedad concreta distribuye fragmentadamente a los individuos, en virtud de la similitud y diferencia de rasgos relacionados, en primer lugar y esencialmente, con la forma en que esa sociedad se reproduce materialmente a sí misma.

Así, la estructura social implica una jerarquía de posiciones sociales (derivada de los roles en la esfera de la producción), un sistema de relaciones sociales entre dichas posiciones y un determinado grado de cercanía y desigualdad entre las posiciones.

Por considerar que las relaciones de propiedad desempeñan el papel determinante en la conformación de las estructuras sociales muchos autores toman las clases (dimanadas de la presencia o ausencia de propiedad sobre los medios de producción) como los elementos esenciales de dicha estructura e identifican la estructura socioclasista como el núcleo de la estructura social, llegando en ocasiones a hacer equival entes ambas categorías. Equivalencia que no significa identidad, sino que denota la centralidad de lo «socioclasista» en la formación de la armazón social y las desigualdades. Sin espacio para adentrarnos en la interesante polémica que sobre este tema se ha mantenido viva en el seno de la sociología, desde sus inicios hasta la actualidad, y para argumen- 
tar la posición de la autora, el centro del análisis recaerá sobre la composición socioclasista de la sociedad cubana y sus cambios en diferentes momentos históricos.

Los cimientos «objetivos» de la estructura y la desigualdad se asocian a la visión de posiciones sociales y las direcciones de movilidad individual deseables, sobre la cima y la sima de dicha estructura y las maneras de llegar a su cúspide. Estas construcciones sociales a su vez acompañan a los factores externos (aquellos que dimanan del modo de producción y de las relaciones sociales que se establecen a nivel de la esfera productiva) en el proceso de configuración de los sistemas de estratificación social.

Estas consideraciones preliminares nos ayudan a comprender que precisamente examinar el estado de la estructura social en un momento dado y las tendencias de su evolución en determinados períodos es un paso inicial, ineludible y esencial para caracterizar cualquier sociedad y evaluar la calidad de las relaciones sociales que le son inherentes.

D esde este punto de mira el texto aborda, en apretadísima síntesis, dos intervalos de la reproducción socioestructural en Cuba: las modificaciones provenientes de la transición social ista y la remodelación asociada a la crisis de los noventa.

\section{Transición socialista y dinámica de los procesos socioestructurales}

Valorar la intensidad y naturaleza de los cambios que tienen lugar en el plano de la composición y las relaciones socioclasistas en una sociedad y un período concretos, exige la consideración de un punto anterior que cumpla el papel de referente histórico de dichos cambios.

Para entender el carácter de las tranformaciones en la estructura clasista cubana como consecuencia de la puesta en práctica de un proyecto económico y social socialista, es necesario ubicar la situación socioestructural típica para los años anteriores al establecimiento de dicho proyecto.

$\mathrm{N}$ umerosos análisis sobre esta época coinciden en líneas generales al definir la estructura socioclasista cubana hacia los años cuarenta y cincuenta². En síntesis, esto puede representarse de la manera siguiente:

- latifundistas;

- burguesía industrial azucarera;

- gran burguesía comercial importadora;

2. Puede consultarse: D IAZ, A. (1988). «La estructura socioclasista de la sociedad cubana». En Conferencia Teórica Internacional «La estructura de clase en América Latina». M emorias. La $\mathrm{H}$ abana.

Fun G, T. (1982). En torno a las regularidadesy particularidades de la Revolución Socialista en Cuba. La H abana: Ed. de Ciencias Sociales.

Rodríguez, C.R. (1979). Cuba en el tránsito al socialismo. 1959-1963. La H abana: Ed. Política. 
- burguesía agraria;

- pequeña burguesía: urbana, rural;

- intelectuales;

- empleados;

- clase obrera;

- campesinos.

Entre los rasgos básicos de esta estructura socioclasista (típica del capitalismo subdesarrollado y dependiente) sobresalen: el bajo peso de la clase obrera, su escasa calificación y preparación profesional y el carácter semiproletario de muchos de sus grupos; la proporción relativamente alta de la pequeña burguesía y los sectores informales; exigua presencia de la intelectualidad y ausencia casi total de imbricación entre el sistema educacional con las necesidades de formación de fuerza de trabajo calificada para un potencial proyecto económico nacional; alto peso del desempleo y marginalismo; predominio casi absoluto de los sectores burgueses no interesados en una vía de desarrollo capitalista independiente, o lo que es igual inexistencia (o bajísima presencia) de una burguesía auténticamente nacional, al to peso del campesinado pobre, no poseedor de la tierra y semiproletario.

Puede apreciarse que el establecimiento de relaciones socialistas de producción en el caso de Cuba implicó prácticamente la destrucción de la estructura socioclasista precedente y su sustitución por una de natural eza totalmente nueva y el predominio casi absoluto de la emergencia de componentes socioclasistas anteriormente inexistentes o de una presencia muy limitada. Ésta es una característica asociada al hecho de que la distancia cualitativa entre la estructura socioclasista propia de un sistema capital ista y la que caracteriza al período de transición al socialismo es mucho mayor desde las condiciones del subdesarrollo.

En tales condiciones se acentúa la complejidad de los cambios socioestructurales, se intensifican los procesos de movilidad social en todos los órdenes y una parte significativa de los diferentes grupos sociales son de «primera generación», carentes de experiencia anterior y las más de las veces de la preparación necesaria, para el desempeño de los nuevos roles económicos y sociales.

Como característica propia de la situación cubana se observa un fuerte y acelerado ritmo de liquidación de la vieja estructura, mucho más acelerado que en otros países que construyeron el socialismo, mientras que las tareas de reproducción estable y elevación sistemática de la eficacia de los componentes de la nueva composición socioestructural transcurren en un ritmo mucho más lento.

Examinando el peso de la producción del sector socialista estatal y cooperativo en diferentes países hacia la segunda mitad de la década de 1960 (a más

3. Tomado de: Burguete, R. (1976). La teoría marxista de las clases sociales y la estructura de la sociedad contemporánea. La H abana: Ed. Ciencias Sociales. 
de dos décadas de socialismo en la mayoría de ellos), comprobamos que la producción industrial privada conservaba el $1,4 \%$ en $H$ ungría, el $2,1 \%$ en la RD A; la de la agricultura el 5,6\% en Checoslovaquia, el 2,4\% en $\mathrm{H}$ ungría, el $88 \%$ en Polonia, el $6 \%$ en la RD A y el $8,8 \%$ en Rumanía, y en comercio minorista la propiedad no estatal representaba el $1,3 \%$ en Polonia y el $21,6 \%$ en la RD A ${ }^{3}$.

En Cuba por estos años y a menos de un quinquenio de revolución, el Estado controlaba casi el $100 \%$ de la industria y el comercio minorista y el $70 \%$ de la producción agrícola 4 .

En la RD A, en el año 1985, los artesanos y comerciantes privados representaban alrededor del 3\% en la estructura clasista de la población y existía también el grupo de los artesanos cooperativistas urbanos que alcanzaba cerca de un $2 \%{ }^{5}$.

Examinando las medidas político-económicas de mayor vínculo con los procesos destructivos y constructivos en el ámbito de la composición y las relaciones de clase en Cuba, tenemos lo siguiente:

- Entre 1959 y 1960 tienen lugar la primera Ley de Reforma Agraria y la nacionalización de las grandes empresas industriales, comerciales, bancarias, etc., medidas que hicieron desaparecer las bases económicas de la gran burguesía nacional y extranjera, iniciaron la formación de un sector de propiedad estatal y en breve lapso limitaron el marco de acción de las relaciones capitalistas a la burguesía agraria media y a la pequeña burguesía urbana y rural.

- Entre finales de 1960 y 1963 se intensifica el desmantelamiento de la peque ña propiedad privada urbana y se pone en práctica la segunda Ley de R eforma Agraria, eliminando así la presencia de la burguesía como tal.

- En 1968 se elimina la pequeña propiedad privada urbana, admitiéndose sólo su presencia en el sector del transporte.

Es palpable que en la primera década de Revolución los procesos liquidacionistas se deselvolvieron a un vertiginoso ritmo y quedaron prácticamente concluidos y que es éste un período donde los cambios socioestructurales tuvieron su acento fundamental en la transformación radical del cuadro clasista de la sociedad cubana.

O bviamente lo que estamos calificando como «proceso liquidacionista», de desmantelamiento de la estructura socioclasista inherente al capitalismo dependiente cubano, lleva implícito su contrario: la formación de una nueva estructura si bien, como antes se señaló, ambas aristas de la transformación socioestructural se desplegaron aceleradamente en el caso de Cuba, la consolidación, la reproducción sistemática y el perfeccionamiento cualitativo de

4. Tomado de: M ARTínez, A. (1964). «El plan de la economía nacional». En revista Cuba Socialista, núm. 31. La H abana.

5. Tomado de: W EIDIG, R. (1988). La estructura social en la RD A. Berlín: D ietz Verlog (en alemán). 
la nueva estructura constituyen fenómenos de elevada complejidad, especialmente para un país subdesarrollado, y por lo tanto requieren de un período más prolongado para su realización.

A finales de los años sesenta quedaron conformados en sus caracteres esenciales los componentes socioclasistas fundamentales de la etapa de tránsito al socialismo: la clase obrera, la capa de los trabajadores intelectuales y la clase del campesinado, acompañadas por una pequeña capa de trabajadores no estatales urbanos, capa de una proporción (al menos formal) mucho más reducida en Cuba que en otros países social istas. En la segunda mitad de los setenta la cooperativización en el campo hace emerger el grupo de los cooperativistas agropecuarios completando el cuadro socioestructural socialista cubano.

Los procesos de naturaleza constructiva han estado fundamentalmente asociados a la conformación del sector de propiedad estatal y la puesta en práctica de diferentes estrategias de desarrollo económico, elementos que han implicado una progresiva complejización de la división social del trabajo y una ampliación del universo socioprofesional y con ello la formación y el crecimiento de nuevos grupos sociales al interior de la clase obrera, la intelectualidad y el campesinado.

Así, las tranformaciones socioclasistas propias de la transición socialista cubana pueden ser divididas en dos grandes períodos: uno - aproximadamente entre 1959 y 1975-, donde se producen los cambios clasistas fundamentales, se desmantelan las relaciones de clases anteriores y se construye un nuevo sistema de componentes socioestructurales y de nexos entre ellos; otro - entre 1976 y 1988-, en el que los nuevos componentes socioestructurales se reproducen cuantitativamente de forma estable y los cambios más intensos se trasladan a nivel de la estructura interna de esos componentes intentando adecuar su estructura profesional y de calificación a los diversos perfiles ocupacionales que han emergido. La tabla 1 nos muestra algunas de estas tendencias reproductivas.

U na mirada rápida a esta última fase, antesala de la crisis de los noventa, permite encontrar un conjunto de interesantísimos rasgos socioestructurales:

1. Fuerte predominio de la estatalización como base de los cambios soci oestructurales

En general, esta tendencia o rasgo cuantitativo tiene un carácter positivo, ya que expresa que el crecimiento de la ocupación implicó un fortalecimiento numérico de la propiedad estatal y que ésta constituyó la fórmula principal para garantizar el empleo a la escala de mayor amplitud posible, lo cual coincide con las direcciones que el modelo de funcionamiento de las relaciones socialistas de producción seleccionado para el período estudiado exigió del proceso de reproducción de la estructura socioclasista.

Pero este ajuste general entre lo real y lo necesario llevó aparejado contradicciones de un carácter más particular, como por ejemplo el crecimiento exce- 
Tabla 1. Cuba - Estructura socioclasista. (D el total de ocupados en la economía nacional. En porcentaje)

Total ocupados (1970)

\begin{tabular}{|c|c|c|}
\hline $\begin{array}{l}\text { Sector estatal } \\
\text { C lase obrera } \\
\text { - O breros producción } \\
\text { - O breros servicios } \\
\text { Administrativos } \\
\text { T écnicos } \\
\text { Dirigentes }\end{array}$ & $\begin{array}{r}45,1 \\
25,3 \\
3,8 \\
9 \\
4,2\end{array}$ & 87,4 \\
\hline $\begin{array}{l}\text { En el sector no estatal } \\
\text { C ampesinado } \\
\text { - Peq. agr. indiv. } \\
\text { Trabajadores } \\
\text { por cuenta propia }\end{array}$ & $\begin{array}{l}9,8 \\
2,8\end{array}$ & 12,6 \\
\hline Total ocupados (1981) & & \\
\hline $\begin{array}{l}\text { Sector estatal } \\
\text { C lase obrera } \\
\text { - O breros producción } \\
\text { - O breros servicios } \\
\text { Administrativos } \\
\text { Técnicos } \\
\text { D irigentes }\end{array}$ & $\begin{array}{r}47,5 \\
12,3 \\
6,7 \\
17,7 \\
7,6\end{array}$ & 91,8 \\
\hline $\begin{array}{l}\text { En el sector no estatal } \\
\text { C ampesinado } \\
\text { - Peq. agr. indiv. } \\
\text { - Cooperativistas } \\
\text { Trabajadores } \\
\text { por cuenta propia }\end{array}$ & $\begin{array}{l}4,3 \\
1,1\end{array}$ & 8,2 \\
\hline Total ocupados (1988) & & \\
\hline $\begin{array}{l}\text { Sector estatal } \\
\text { C lase obrera } \\
\text { - O breros producción } \\
\text { - O breros servicios } \\
\text { Administrativos } \\
\text { Técnicos } \\
\text { D irigentes }\end{array}$ & $\begin{array}{r}48 \\
13 \\
6,5 \\
20,1 \\
6,4\end{array}$ & 94 \\
\hline $\begin{array}{l}\text { En el sector no estatal } \\
\text { C ampesinado } \\
\text { - Peq. agr. indiv. } \\
\text { - Cooperativistas } \\
\text { Trabajadores } \\
\text { por cuenta propia }\end{array}$ & $\begin{array}{l}3 \\
2\end{array}$ & 6 \\
\hline
\end{tabular}

Fuentes: JU CEPLAN. Boletín Estadístico de 1970. Com ité Estatal de Estadística. Censos de Población y Vivienda 1970 y 1981. Com Ité EstatAL DE Estadíst ICA. Anuario Estadístico de Cuba, 1988. 
sivo y desproporcionado del grupo de los empleados administrativos, los dirigentes y especialistas ocupados en actividades de la planificación y el control, lo que significó un deterioro de la proporción entre los trabajadores directamente vinculados a la producción y los servicios y los improductivos, y puso en evidencia síntomas de burocratización en el sistema de organización de la sociedad.

Aunque a mediados de los años setenta se extiende la propiedad cooperativa agropecuaria y se conserva un sector relativamente escaso de pequeños agricultores privados y trabajadores por cuenta propia urbanos, el peso del sector estatal es débil y predominan en la política socioeconómica las medidas destructivas de la pequeña producción privada urbana, a la que, como peculiaridad de la transición socialista cubana, no se le concedía un espacio de complementación de las relaciones socialistas de producción.

Por otra parte, no siempre el crecimiento de la ocupación en el sector estatal se vio acompañado de la elevación de la productividad del trabajo y del mejoramiento de los indicadores de calidad de la producción y los servicios.

D e igual modo, la clase obrera, aunque creció en términos absolutos, disminuyó su peso relativo en el conjunto de la estructura socioclasista, bajo los efectos de la fuerte aceleración del ritmo de crecimiento de la capa de los trabajadores intelectuales.

En síntesis, puede decirse que hasta los años ochenta el modelo de organización de las relaciones de propiedad sobre los medios de producción adoptado en Cuba identificó, de hecho, propiedad socialista con estatalización, lo que impidió el despliegue de otras formas de propiedad - que pueden ser también socialistas 0 al menos no representan un obstáculo real para el desarrollo de las que tienen ese carácter - , sobrecargando al Estado con un sin número de funciones económicas y administrativas y haciéndolo responsable casi absoluto de proveer fuentes de empleo e ingresos estables a la gran mayoría de la población.

2. Elevado grado de diversificación y heterogeneidad de la estructura soci oclasista que se intensificaba progresivamente

Esta diversidad se expresó en:

- Presencia de tres formas diferentes de propiedad sobre los medios de producción estatal, cooperativa y privada (pequeña urbana y rural). Aún dentro del amplio predominio de la estatal.

- Gran variedad de grupos socioprofesionales en consonancia con la complejización de la división social del trabajo.

- H eterogeneidad de grupos por calificación.

- Amplio espectro de grupos salariales.

- Distancia cualitativa relativamente grande entre los grupos extremos según propiedad, situación socioprofesional, calificación eingresos, en general y en el interior de cada componente. 
La complejidad y variedad de la estructura sociocl asista que expresan estos elementos, es un efecto lógico de la diversificación económica, que llevó implícita la multiplicación de ocupaciones, y de que el desarrollo de la base técnicomaterial no marchó de forma pareja en todas las ramas y actividades laborales, lo que generó la aparición y el incremento de grupos avanzados en la estructura interna de los diferentes componentes socioclasistas (en el sentido de las exigencias del carácter y el contenido del trabajo, la calificación y sus condiciones materiales) y la supervivencia de grupos retrasados, extremos que constituyen polos entre los cuales se estructura todo un conjunto de otros grupos que expre san diferentes grados de desarrollo de los rasgos de la ubicación socioprofesional.

\section{Insuficiente fortaleza de los núcleos centrales de los componentes sociodasistas fundamentales}

Tendencias tales como la disminución acelerada de los obreros agrícolas ${ }^{6}$, el crecimiento excesivo de grupos no vinculados a la producción material, la débil presencia de la intelectualidad técnico-ingenieril, la disminución e insuficiente eficacia productiva del grupo de los campesinos cooperativistas, junto al fortalecimiento de los pequeños agricultores individuales, explican por sí solas este rasgo general y señalan claramente que en el período analizado aparecieron serios obstáculos para el desarrollo de componentes esenciales de la estructura social, sugiriendo la necesidad de revisar las estrategias económicas y sociales diseñadas y su puesta en práctica.

4. Predominio de los procesos de movilidad intraclasista (a nivel de estructura interna)

Entre los elementos más importantes asociados a este predominio pueden señalarse:

- Aumento de la movilidad a medida que se desciende en el nivel de generalidad de la diferenciación socioestructural: los grupos formados por el criterio de propiedad fueron mucho más estables que los socioprofesionales.

- Baja presencia de los desplazamientos sociales que implican «salto» 0 cambio brusco de la ubicación socioestructural.

6. Aunque a escala mundial, en los países avanzados la disminución de los obreros agrícolas puede ser tomado como un indicador de progreso, en el caso de Cuba, país de fuerte base agropecuaria, esta tendencia no fue un resultado directo del crecimiento de la productividad en este sector sino del efecto combinado de la fuerza de atracción que el proceso de urbanización ejerció sobre los trabajadores del campo, despoblando importantes zonas agrarias, y de la elevación de la producción sobre la base de altos costos en insumos. 
- Predominio de los desplazamientos que significaron movimiento entre grupos cercanos (atendiendo al carácter y contenido de la actividad laboral) y conservación de los rasgos más fuertes de la pertenencia socioclasista.

- Presencia de incipientes corrientes de movilidad hacia la propiedad no estatal desde la clase obrera y los trabajadores intel ectuales.

- Predominio de la movilidad como factor de crecimiento de la capa de los trabajadores no estatales.

- Estabilidad y crecimiento a partir de la movilidad social de los vinculados al sector industrial.

Se evidenciaron claramente además problemas que denotan la pérdida de potencialidades progresivas de la movilidad social:

- M ovilidad social como causa de decrecimiento del grupo de los obreros directamente vinculados a la producción (especialmente agropecuarios y de la construcción) y de incremento de grupos no fundamentales de la clase obrera (trabajadores de los servicios).

- Agotamiento de las posibilidades reales de ascenso socioeconómico para muchos grupos sociales.

- Autorreproducción residual de la clase obrera.

\section{Existencia e intensificación de los ritmos de reproducción} de una estructura socioclasista paralela

Este rasgo significa que existen elementos de la estructura social que tienen un doble rol, de posiciones antagónicas, en el funcionamiento de la economía nacional, uno de ellos generados por su ubicación en el sistema de relaciones socioeconómicas propias del model o formal de funcionamiento del modo de producción socialista en Cuba y el otro por su inclusión en una economía sumergida, que implica la negación del funcionamiento de la propiedad social y su utilización con fines privados.

Entre sus cau sas básicas pueden apuntarse: la existencia de amplias franjas de necesidades no cubiertas, descontrol sobre los bienes sociales e inadecuado tratamiento a la pequeña producción privada.

En su conjunto estos rasgos dibujan un panorama de el evadísima complejidad socioestructural que constituye el antecedente directo de la aún más compleja década de los noventa.

\section{La etapa actual: la crisis, el reajuste económico y el nuevo modelo para la estructura social}

D esde finales de los ochenta e inicios de los noventa podemos reconocer la presencia de una crisis en la sociedad cubana de natural eza estructural - no coyuntural - dada por el hecho de que la armazón tradicional de su funcionamiento dejó de garantizar la reproducción ampliada del sistema socioeco- 
nómico en su conjunto y la respuesta adecuada a los nuevos requerimientos internos y externos de esa reproducción?.

Los elementos básicos de esta crisis no son de reciente aparición, sino que comenzaron a dejarse sentir desde mediados de los años ochenta, en problemas tales como el decrecimiento de la productividad, la ausencia de correspondencia entre gastos sociales y resultados económicos, exceso de liquidez y desequilibrio de las finanzas internas, crecimiento del mercado negro y la economía sumergida, inicio de la caída del salario real, crecimiento del subempleo, absorción ineficiente de los incrementos de la población económicamente activa por el sector estatal, insuficiente despliegue y fortal ecimiento de las formas de propiedad socialista, debilitamiento de los núcleos centrales de los componentes socioclasistas fundamentales, situación relativamente desventajosa de la clase obrera, entre otros, síntomas que daban claras señales de que el modelo económico adoptado se hacía inviable.

La desaparición de la comunidad socialista europea y de la U RSS y el recrudecimiento del bloqueo económico norteamericano hacia Cuba, lógicamente agudizaron estos elementos, fueron su detonante y a partir de ese momento se produjo la caída sistemática de la actividad económica y se hizo imposible sostener una lógica de enfrentamiento a la crisis lenta, moderada y conservadora.

El conjunto de medidas que conforman el proceso de reajuste ante la crisis, ha generado una nueva fase en el funcionamiento de las relaciones económicas al basarse en un cambio en las relaciones de producción, que llega incluso a implicar una modificación del sistema de propiedad. Esta fase puede ser considerada como de transición desde un modelo de socialismo hacia un nuevo sistema socioeconómico que combina elementos del socialismo con una economía de mercado ${ }^{8}$.

Reconocer la etapa actual como de crisis y transición y como modificación de las relaciones de propiedad, es de importancia vital para comprender la evolución socioestructural, pues ella depende en grado sumo de la naturaleza de la estructura económica. A priori puede afirmarse entonces que la estructura socioclasista cubana también expresa la presencia de la crisis y debe transitar hacia un nuevo modelo, como efecto del cambio económico y como dinamizador y prerrequisito de éste.

Las direcciones fundamentales que dan cuerpo a la transición y adelantan algunas características del nuevo modelo que va configurándose son las siguientes 9 :

7. Ver, por ejemplo: García VALDÉs, C. (1994). La economía cubana en los 90: transformaciones en el modelo socialista. La H abana: IN IE.

Carranza, J. (1995). «Los cambios económicos en Cuba: problemas y desafíos». En Dossier 819. La H abana: CEA.

8. Ver por ejemplo: Ferrer, P. (1994). «El enfoque social de la economía cubana en 1994. N uevos escenarios y retos». La H abana: Centro de Estudios de la Economía Cubana.

García Valdés, C. O p. cit.

9. Consultar: CARRANZA, J. O p. cit.

García VALDÉs, C. Op. cit. 
- Diversificación y redimensionamiento de las formas de propiedad: aparición del sector de economía mixta, ampliación de la pequeña producción mercantil urbana y rural, extensión del sector cooperativo agropecuario, decrecimiento del sector estatal.

- Modificación del papel del Estado en la economía: aumento del grado de descentralización y autonomía en la toma de decisiones, ampliación del rol de los mecanismos de mercado y potenciación de la planificación estra tégica, pérdida del monopolio estatal sobre el comercio exterior, potenciación de los instrumentos de control monetario.

- Reforma empresarial: reconversión industrial y tecnológica, cambios en los principios organizativos de la producción en los estilos de gerencia, cambios en las formas de estimulación del trabajo.

- Reestructuración de las formas de empleo y las fuentes de ingresos.

- Potenciación de sectores económicos no tradicionales, como el turismo y la biotecnología.

- Adopción de un nuevo sistema de control de las finanzas internas: incremento de precios y creación de un mecanismo impositivo tributario que responden a la magnitud y a las formas de los ingresos en los diferentes grupos sociales.

- Liberalización de las divisas y dualidad monetaria interna.

Ellos deben complementarse con otros elementos que forman lo que podría llamarse un «escenario activo futuro»:

- Estructuración sistemática del conjunto de medidas económicas que asegure el papel rector de una estrategia integral, que armonice la lógica del funcionamiento económico y los intereses del progreso social.

- Radicalización de las medidas de carácter financiero que recuperen el valor de la moneda nacional, del salario real y del sistema de estimulación al trabajo. Estas acciones deben orientarse al mejoramiento de la situación de los grupos cuyos ingresos provienen del trabajo, especialmente los vinculados al sector estatal.

- Perfeccionamiento integral del sistema empresarial orientado a crear una empresa estatal autónoma de carácter mercantil competitiva y eficiente. Este logro de la eficacia empresarial constituye uno de los pivotes para garantizar el carácter hegemónico y rector de la propiedad socialista y de los grupos a ella asociadas, dentro del sistema multisectorial de propiedad existente en el país que tiende a ampliarse.

- D escentralización de la política de empleo orientada hacia: potenciación del papel de los territorios y las localidades en la identificación de las fuentes de empleo, plena apertura del trabajo por cuenta propia, incluyendo la posibilidad de creación de cooperativas y microempresas privadas en actividades como el comercio minorista, servicios y gastronomía y pequeñas producciones para mercados reducidos, liberalización del mercado de trabajo en sectores que así lo requieran, como el caso de la agricultura. 
- Debilitamiento del rol de la economía sumergida.

Reflexionando sobre los factores de cambio económico ya actuantes y sus posibles escenarios futuros, salta a la vista, como se ha venido señalando, que uno de sus primeros efectos sobre la vida de la sociedad recae en la distribución por estratos sociales y su jerarquía de acuerdo a las funciones económicas que les competen y el grado de desigualdad que le es inherente a esta estratificación.

Si seleccionamos algunos factores básicos asociados a las reproducción socioestructural, como por ejemplo el régimen de propiedad, los niveles de desarrollo tecnológico, el sistema de formación de fuerza de trabajo calificada, el empleo, los ingresos, la dinámica poblacional y la imagen subjetiva de la estratificación social, comprobamos sin dificultad la apertura de una nueva fase en dicha reproducción. Entre los contenidos clave de estos factores que caracterizan esta nueva etapa podemos situar los siguientes:

- Sistema de propiedad multisectorial amplio con disminución del peso de la propiedad estatal a favor del crecimiento de la propiedad cooperativa, mixta y privada. Carácter hegemónico de la propiedad estatal y concesión de un espacio de complementación y de funciones relevantes en la producción y los servicios de otras formas de propiedad que puede ampliarse progresivamente. A diferencia de los períodos anteriores. La convivencia de formas de propiedad diferentes no es considerada un remanente a eliminar, sino un rasgo consustancial del funcionamiento de la economía que posibilita la ampliación y el desarrollo de ésta. N o es un factor coyuntural sino de funcionamiento a largo plazo.

- Fortalecimiento de la dispersión tecnológica, caracterizada por la convivencia de procesos de utilización de alta tecnología y junto a los de regresión tecnológica.

- Tendencia a la descentralización progresiva de las estrategias de empleo priorizando las necesidades y las capacidades a nivel sectorial y territorial. Elevada capacidad empleadora de la propiedad no estatal y de la economía informal. Imposibilidad de garantizar el pleno empleo a cuenta del sector estatal. M ayor peso de los criterios de eficiencia en la política ocupacional y orientación hacia la eliminación del subempleo. Priorización del sector agropecuario, turismo, industria azucarera y médico-farmacéutica.

- Tendencia al fortalecimiento de la descentralización sectorial y territorial en el diseño de la política educacional con restricciones y disminución del acceso en sus niveles de formación profesional, particularmente el superior. Priorización de la formación de técnicos medios y obreros calificados en ocupaciones vinculadas a actividades estratégicas con perfiles ocupacionales amplios.

- Tendencia a la descentralización de las políticas de estimulación a nivel sectorial, ramal y territorial. Fortalecimiento de su vínculo con la eficiencia económica real y de su carácter diferenciador entre grupos profesionales. 
Alta diversificación en la fuente y en la magnitud de los ingresos, fuerte peso en éstos de fuentes no relacionadas con el salario y con el trabajo.

- Fortalecimiento de la tendencia al envejecimiento de la población. Elevación de la incidencia de la migración exterior. Disminución, aunque aún débil, de la migración campo-cuidad e incipientes corrientes de retorno a las áreas agrícolas.

- Percepción de las ocupaciones que permiten el acceso a la divisa y a altos niveles de consumo como cima de la posición social, al margen de los reque rimientos de instrucción y calificación y de la correlación entre trabajo manual e intelectual. 0 btención de divisas y alto consumo como criterios de prestigio social y como modelo subjetivo de movilidad ascendente.

- Percepción de un alto grado de desigualdad social no siempre asociado al trabajo, que puede ser fuente de conflicto sociopolítico.

- Elevado dinamismo de la movilidad social ascendente y descendente, desde el punto de vista del bienestar económico que incluye la desestatalización de los núcleos centrales de las clase y capas sociales. Apertura de nuevos canales de ascenso que coexisten con fuertes corrientes de descenso como reflejo de la ampliación de las desigualdades sociales.

En síntesis, este cuadro muestra una clara disminución de la intervención del Estado en el diseño y en la reproducción de la estructura social y el paso hacia mecanismos reproductivos más diversos, flexibles y complejos que si bien deben funcionar de manera complementaria para asegurar la formación y estabilidad de la estructura social necesaria a la estrategia económica, pueden en la práctica actuar de forma contradictoria.

Q uizás el signo que con mayor claridad evidencia la presencia de una nueva etapa en la estructura es precisamente la potenciación de la diferenciación socioeconómica y la desigualdad social, que han multiplicado los grupos diferentes e incluso han hecho aparecer segmentos sociales con algunos rasgos opuestos y han aumentado considerablemente la distancia social entre los grupos.

La tabla 2 nos muestra, en términos cuantitativos, como las modificaciones en los factores anteriormente reseñados han dado lugar a cambios radicales en la estructura socioclasista de la sociedad cubana actual.

El tipo socioestructural anterior, típico de la transición socialista cubana, comenzó a gestarse desde el mismo inicio de la revolución y pasó por diferentes fases que representaban niveles superiores de profundización en una misma lógica reproductiva, con predominio de los procesos de continuidad sobre los de cambio, el avance hacia grados cada vez superiores en la eliminación de las desigualdades sociales, el acercamiento entre los diferentes componentes socioestructurales, la desaparición paulatina de las clases sociales, la ampliación sistemática de la integración social y la supresión prácticamente total de espacios de exclusión (en el sentido de lograr un acceso igualitario al bienestar material y espiritual para todos los grupos sociales).

Al margen de que en esta lógica quedó fuera una adecuada comprensión del rol de las diferencias socioeconómicas necesarias como una de las fuentes 
Tabla 2. Cuba 1994 - Estructura socioclasista. (D el total de ocupados en la economía nacional. En porcentaje)

$\begin{array}{lc}\text { Sector estatal } & \\ \text { Clase obrera } & 42,1 \\ \text { Administrativos } & 4 \\ \text { Técnicos } & 17,5 \\ \text { Dirigentes } & 5,7\end{array}$

69,2

Sector de propiedad mixta

Campesinado

Peq. agr. indiv. $\quad 3,3$

Cooperativistas $\quad 7,6$

Trabajadores no estatales (por cuenta propia)

del progreso en el socialismo y se absolutizó el papel de la igualdad, con lo que se asumió una política distributiva igualitarista, en buena medida desconectada de un imprescindible nexo con la eficacia productiva, es innegable que se obtuvieron reales avances en el llamado proceso de «homogeneidad social», de superación de diferencias y exclusiones injustas y en la creación de una estructura con una distancia cualitativa relativamente pequeña entre sus grupos extremos (los más avanzados y los más rezagados) si la comparamos con cualquier otra estructura nacional en los años ochenta. En ese contexto, las diferencias socioeconómicas intergrupales no tenían una equivalencia directa en la desigualdad social.

La inviabilidad del modelo económico, al estar asociada, entre otros factores, a un estancamiento de las relaciones de producción y dentro de ellas al sistema de propiedad, convierte también en inviable el modelo de estructura socioclasista con que había funcionado y reclama un cambio radical en este sentido.

D esde nuestra óptica, la estrategia económica modelada como respuesta a la crisis ha perfilado como elementos poseedores de una alta capacidad estructuradora de grupos sociales y generadora de desigualdades a la presencia creciente de la propiedad mixta y las inversiones del capital extranjero, la parcelación y entrega en usufructo a productores individuales, familiares o cooperados de tierras de propiedad estatal, la ampliación de la pequeña producción y los servicios en el sector privado urbano, la aceleradísima expansión del turismo, el impulso a las actividades biotecnológicas (como franja de al ta tecnología) y la diversificación de las fuentes y la magnitud de los ingresos ${ }^{10}$. M . y otros (1995). I mpactos socioestructurales del reajuste económico. Fondos del CIPS. L a H abana. Informe de investigación. 
El nuevo tipo de estructura que emerge impulsada por el reordenamiento económico, supone la aparición de procesos de ruptura con relación a su antecesor y el predominio del cambio sobre la continuidad. Comparando la actual composición socioclasista de la sociedad cubana con la situación anterior a los años noventa, es posible distinguir algunos de los rasgos fundamentales que caracterizan a este nuevo tipo socioestructural:

1. Diversificación acelerada de las formas de propiedad.

2. H eterogeneización y complejidad crecientes. Fuerte diferenciación socioe conómica general y fragmentación de las estructuras internas de las clases y de las capas sociales.

3. Altos grados de desigualdad social que se expresa fundamentalmente en:

- Aumento progresivo de la distancia cualitativa entre los grupos extremos. En este sentido, aparición de «élites» y «vulnerables».

- Acceso selectivo y restringido a los más altos niveles de consumo y el bienestar material.

4. Presencia de fuertes desigualdades no asociadas al trabajo y al rol de cada grupo en la estrategia socioeconómica.

5. Fortalecimiento de los vínculos entre formación y crecimiento de grupos sociales y la eficacia productiva de éstos.

6. Elevada movilidad social que tiene entre sus direcciones más fuertes el paso de la propiedad estatal a la no estatal y del sector tradicional de la economía al emergente.

Ante la constatación de estos rasgos, la primera pregunta que surge es si ellos representan procesos positivos o negativos en la evolución socioestructural y un grado superior o inferior, regresivo o progresivo en este proceso. La respuesta no es nada simple y exige una valoración muy mesurada, es más, probablemente no exista una respuesta única y ella dependa de la óptica evaluativa que adoptemos.

$\mathrm{Si}$, evidentemente, a mediados de los ochenta aparecieron ya claros vestigios de la «inviabilidad del modelo socioestructural», como por ejemplo el debilitamiento de los núcleos centrales de los componentes básicos, el estancamiento de la movilidad social ascendente, la desconexión entre el crecimiento de grupos sociales y su efectividad productiva, la ampliación de la estructura social sumergida, entre otros, es innegable que tal estructura debía ser sustituida.

Cambiar el diseño del sistema de propiedad implica remodelar radicalmente las relaciones sociales y la armazón estructural que las sostiene.

D entro de este contexto, entonces rasgos como la diversificación de las formas de propiedad, la fuerte diferenciación socioeconómica, la elevada movilidad social, la elevación del rol de la eficacia productiva en la formación de grupos socialese, incluso, la aparición de algunos grupos «élites», significan la solución a viejos problemas y la adecuación a las nuevas circunstancias, donde sin resultados económicos el proyecto sociopolítico nacional no tiene perspectiva. 
Aquí aparece uno de los retos más complicados del actual proceso de transformación: la nueva y diferente estructura socioclasista debe cumplir funciones de su antecesora: propiciar y mantener altos grados de equidad y justicia social. Este reto se encadena con otra interrogante: iA qué tipo de sociedad corresponde esta estructura? ¿Ella se mantiene dentro de una concepción social ista?

El pensamiento teórico socialista y la experiencia práctica de otras naciones de ninguna manera contraponen un diseño estructural multisectorial con fuerte presencia de la propiedad privada, incluido el capital extranjero con una estructura socialista, de manera que no es la cubana una situación inédita.

Por supuesto esta multisectorialidad supone la convivencia de actores socioeconómicos muy disímiles y hasta contrapuestos, para al gunos de los cuales las perspectivas de progreso futuro no necesariamente se vinculan al desarrollo del socialismo, tal y como éste ha sido tradicionalmente entendido, y en la práctica cubana está creando márgenes de desigualdad superiores a los aceptables por este régimen social.

En resumen, ello significa renunciar a un ideal de homogeneidad social y acelerada desaparición de las clases sociales y aceptar un modelo socioestructural de alta diferenciación socioeconómica, como base del desarrollo económico, que atenúa las desigualdades inter e intraclasistas y grupales a través de la redistribución equitativa y de la amplia participación política de los sectores populares.

Pero rasgos tales como la aparición de grupos vulnerables, las desigualdades no asociadas al trabajo y el acceso restringido al bienestar material indican la pre sencia de procesos de exclusión y desintegración que deben ser considerados como profundamente negativos y que deben ser eliminados en una perspectiva no muy larga, pues su consolidación en el tiempo sí afectaría al carácter socialista y al contenido de justicia social del sistema cubano y rompería el consenso político en que éste se ha basado hasta ahora.

Sería un gravísimo error aceptar estos fenómenos como efectos irremediables del funcionamiento de la economía, cuya naturaleza objetiva imposibilita toda acción política correctora.

I gualmente los gérmenes para la formación de una pequeña burguesía alta y media en nuestra sociedad y el rol político de esta posible nueva clase social junto al del capital extranjero, señalan procesos aún no desplegados en toda su real dimensión que es imposible ignorar en su relación con el socialismo posible y su futuro. 